\title{
DEVELOPMENT OF AN ANIMATED VIDEO FOR PRE- AND POSTOPERATIVE LEARNING FOR OSTEOARTHRITIS PATIENTS
}

\author{
Erik Kylén ${ }^{1}$, Joel Stenholm ${ }^{1}$, \& Ann Svensson ${ }^{2,3}$ \\ ${ }^{1}$ MedFilm AB (Sweden) \\ ${ }^{2}$ School of Business, Economics and IT, University West (Sweden) \\ ${ }^{3}$ Health Academy West (Sweden)
}

\begin{abstract}
Osteoarthritis (OA) is a condition in the joint that is developing for a long time and hit first and mainly articular cartilage, and it is a long-term chronic disease characterized by deterioration. In the final stage it is affected all the tissues of the joints such as bones, ligaments, arthritis, joint fluid and muscles, which results in bones rubbing together and creating stiffness, pain, and impaired movement. This disease can affect the joints in the knees, hands, feet, spine and shoulder. OA is the single most common cause of disability in older adults worldwide. Patients need to receive detailed information about the disability, learn about their disease, its treatment and how to follow recommendations both before and after the surgery. As hospital stays are shortening the need of information is increasing, as the patients need to prepare themselves at home before the operation, as well as take care of their rehabilitation when they are coming home, discharged from hospital. In this paper we present a case study about the development of a web-based animated video that was developed to support the learning for patients with OA, using a design science research approach. DSR is an iterative process that include three iterative research cycles. The relevance cycle provided the requirements for the application from the very beginning. The relevance cycle, the rigor cycle and the design cycle were conducted iteratively, and have generated design alternatives that have been evaluated in discussions with OA surgery physicians, as representatives from the application domain.
\end{abstract}

Keywords: Osteoarthritis, animated video, patient learning, surgery, design science research.

\section{Introduction}

Osteoarthritis $(\mathrm{OA})$ is a condition in the hip or knee joints that develops during a long period of time and affects first and mainly articular cartilage. OA is a long-term chronic disease characterized by deterioration. In the final stage it has affected all the tissues of the joints such as bones, ligaments, joint fluid and muscles, which results in bones rubbing together and creating stiffness, pain, and impaired movement. The condition can also affect the joints of hands, feet, spine and shoulders. However, OA is most common in hips and knees. OA is also the single most common cause of disability in older adults worldwide (Palazzo, et al., 2016). It is estimated that 10 to 15 per cent of all adults in the age of 60 and above have some degree of OA. Every tenth man and every fifth woman over 60 are estimated to have $\mathrm{OA}$. The condition is not considered a part of the natural aging process, although, it is more common among elderly. Approximately one in four above the age of 45 in Sweden have OA, and in line with an aging and increasingly obese population, the condition is expected to become even more widespread. This will cause an increased pressure on the healthcare worldwide. However, OA can be treated with physical activity, and anyone with OA can do a lot by themselves to improve their health. It is recommended to continue being active adjusting the activities in accordance with the physical circumstances. Although, many patients still require hip or knee joint replacement surgery when pain gets too in-tense. Studies have demonstrated good outcomes of joint replacement surgery (Hamel, et al., 2008).

Patients require detailed information about their condition and upcoming surgical procedure, in order to comprehend the process of pre- and post-operative recommendations; how to prepare before surgery and how to carry out physio-therapy afterwards. As hospital stays are shortening, the need for information is increasing, as the preparations need to be carried out at home to a greater extent., as well as taking care of their rehabilitation when they return home. The hospitals strive to reduce the number of 
operations canceled, due to unprepared patients, as this entails costs for unused resources such as surgeons, nurses, as well as operating rooms. Not only is patient satisfaction an important criteria for the overall healthcare service quality, but for the society at large

Healthcare professionals giving medical advice orally has not proven to be a successful method for patient compliance. Neither is handing out written information sufficient, as written instructions can be hard to understand. However, animation and cartoons have been used to improve adherence to instructions, which have shown better patient understanding (Kessels, 2003). Digital tools have also been tested, for example video-conferencing to provide tele-rehabilitation services allowing patients to consult with a physiotherapist from their home (Lawford, et al., 2018). The effectiveness of a web-based exercise therapy program tended to improve in physical function of the patients (Uesugi, et al., 2018). However, the digital technologies can be developed to better suit the patients' needs for information and instructions. In this paper we present a case study about the design and development of a web-based 3D-animated video film that was developed to support education for patients suffering from OA, using a design science research approach.

\section{Research method and design process}

The design science research (DSR) approach was followed by creating a web-based animated video. Two of the authors are specialists in 3D-animation and informatics and have been working through the entire design process, from the very beginning in collaboration with healthcare professionals. The third author has been involved in the documentation of the design science research process. The case study presented in this paper is motivated by the desire to improve the patients' learning by the introduction of a new and innovative digital application, and the processes behind this. Thus, DSR is a suitable framework to reach this goal. DSR is an iterative process that includes three iterative research cycles (Hevner, 2007; Hevner, et al., 2004).

The first cycle, the relevance cycle, provided requirements for the application from the very beginning. In this cycle our team consulted OA surgeons and other healthcare professionals such as nurses, assistance nurses and physiotherapists. In the beginning, employees from the hospitals' communication department were included. Three group meetings in total were conducted in order to identify challenges in learning and instructing patients who will be subjected to OA surgery and the following rehabilitation. This cycle also defined the acceptance criteria for the evaluation of the results. The acceptance criteria were defined in a manuscript for the application, with 5 different scenes, each describing «What We See» (Imagery) and «What We Hear» (Dialogue). In each scene 5 to 25 different parts were describing the acceptance criteria.

The relevance cycle was connected to the rigor cycle in iterations, as literature reviews of the knowledge base of the application domain were conducted. Moreover, expertise was consulted to define the state of the art in the domain and processes of OA surgery, together with patients' required preparations and rehabilitation activities.

The iterative design cycle has generated design alternatives that have been evaluated in discussions with OA surgeons and nurses as representatives from the application domain. Staff from the communication department and patients were also included in the iterative design cycle. The evaluating activities were ongoing until a satisfactory design was achieved. Moreover, the evaluations in the design cycle also resulted in additional iterations of the relevance cycle to improve the requirements. This was done in order to increase the application's utility in practice in the OA surgery domain of patients' increased learning. Iterative building and evaluating activities are significant characteristics of DSR framework (Hevner \& Chatterjee, 2010). This is a novel application, useful for teaching patients what to expect and how to prepare in relation to an OA surgery. Therefore, this study can contribute to the design knowledge (Baskerville, et al., 2018).

\section{Results: the rigor cycle}

In order to accomplish the objective to design and develop a 3D-animated film to prepare patients for OA surgery, and to teach patients how to conduct the rehabilitation process, we studied OA literature, literature on technology adoption and 3D-user interfaces. The OA literature informed us on how the hip and knee joints are constructed, why a surgery could be necessary, how the patient needs to prepare, how the operation will be conducted, and how the patient should carry out their rehabilitation. It is crucial to understand all these areas in order to design the application on OA from a patient perspective. We turned to both scientific and popular literature to gain knowledge about the domain. We also had the opportunity to discuss the state of the art in the OA domain with OA surgeons. 


\section{Results: the relevance cycle}

In order to design the application for patients' learning of OA we first analysed the process of pre- and post-operation of OA surgery, together with intrinsic details of OA and its characteristics. To fully understand the requirements and the evaluation criteria of the application we have conducted a thorough ethnographic research with triangulation. Field observations were carried out by two of the authors, attending OA surgery operations at a hospital in Sweden. Interviews with OA surgeons and groups interview were conducted. The observations were captured in field notes afterwards and the interviews were documented in written notes during the interviews. The two group interviews' discussions were based on different versions of the manuscript for the 3D animation. The results were summarized in a new version of the manuscript containing the sequence of the scenes in the $3 \mathrm{D}$ animated video: (1) anatomy, (2) why surgery, (3) before operation, (4) operation, and (5) after operation. 5-25 parts were identified in each of the scenes, as these parts are also defining the evaluation criteria.

The group interviews discussions were conducted in order to get insights in the surgical process including pre- and postoperative activities, and to get input to be able to conceptualize the first version of the prototype for the 3D animated video. The goal of the relevance cycle was to get a detail understanding before creating the first prototype of the $3 \mathrm{D}$ animation as well as the associated requirements and evaluation criteria. Table 1 provides an overview of the operation scene of the first version of the manuscript for the 3D animation, where «what we see» is what is expected to be animated, as not yet analysed, and «what we hear» is what is expected to be told by the spoken narratives in the $3 \mathrm{D}$ animated video.

\section{Results: the design cycle}

Together with our insights from the rigor and relevance cycles, our previous experiences in designing 3D animated videos as well as the technology acceptance literature, we designed two different digital prototypes before the last version of the $3 \mathrm{D}$ animated video was released. However, even after the 3D animated video was implemented, we still received feedback on the final result. We followed a user-centered approach following the design requirements in creating the $3 \mathrm{D}$ animated video in the design cycle.

\subsection{First design cycle - animation exploration}

In the first design cycle we focused on designing the human body as natural as possible in the animated video. We experimented with both a male and a female human body. We especially focused also on the challenge in finding a balance between showing frightening pictures of body parts as well as lifelike pictures of the hip joint, see the design process in figure 1. Insights from the relevance cycle taught us for example to avoid showing blood in the animated video. In the first design cycle we also designed the five scenes with the parts identified in the relevance cycle. In the first design cycle there is also a focus on finding a balance between the time used for showing the animations in relation to the time it takes for the spoken narrative to read the corresponding text.

Figure 1. How it looks like in the workflow in the design process before the images are rendered.

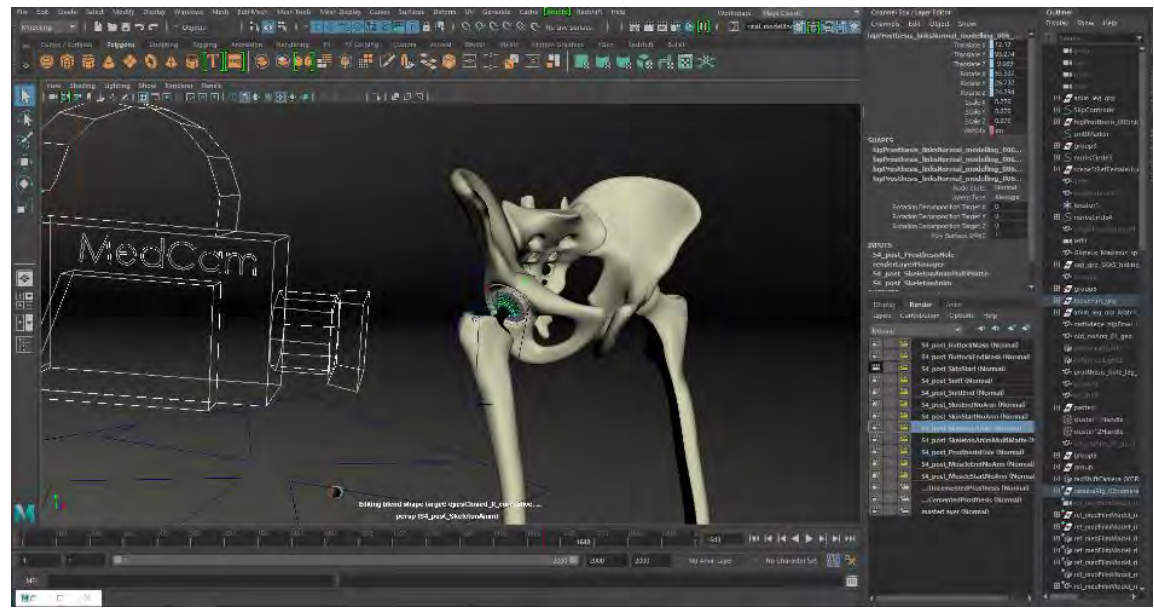




\subsection{Second design cycle - design validation}

In the second design cycle the different parts in the five scenes were revised, in order to customize the content to reflect the specific workflow of the clinic in question. The second design cycle also resulted in removing the scalpel from the animated video, and to not show the incision as it looks like in reality. Instead, the incision was shown as a yellow stripe on the body, as in figure 2 .

Figure 2. The incision shown in the film, that is around 15 centimeters.

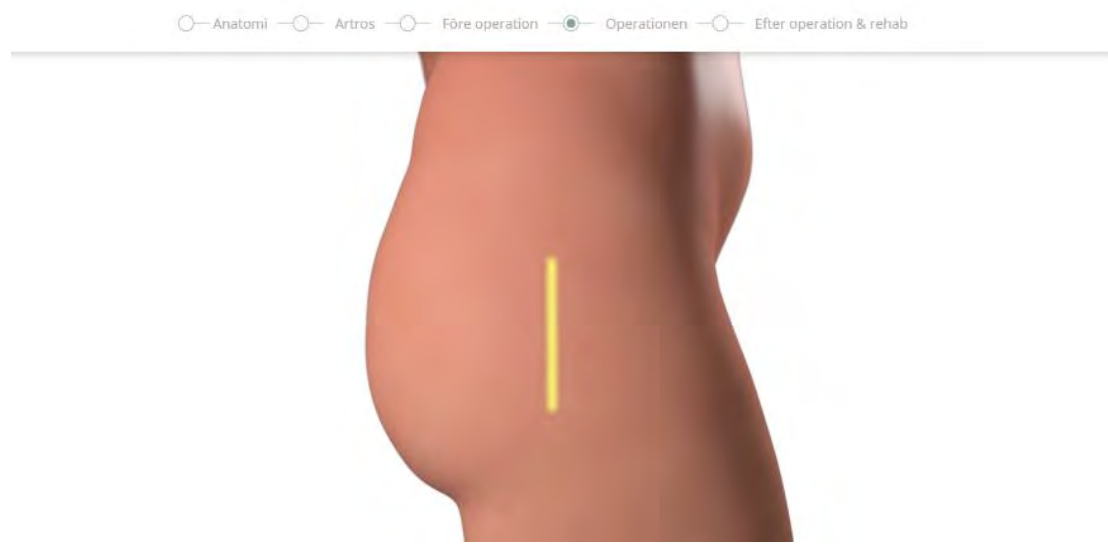

\subsection{Third design cycle - validation after implementation}

The third design cycle is an ongoing cycle after the films have been implemented into the daily routines of the clinic. In this cycle there is a continuous feedback-loop where healthcare personnel and patients from time to time comment on the film content, making the product development a constant process. The two figures, 3 and 4 , show the improvements from a previous version to a later version of the film, showing the moment of the artificial hip component that is fitted to the acetabulum. The developed version shows a more pedagogical view of the hip, and the picture is of a better quality.

Figure 3. First version of the implemented film, showing the artificial hip component that is fitted to the acetabulum.

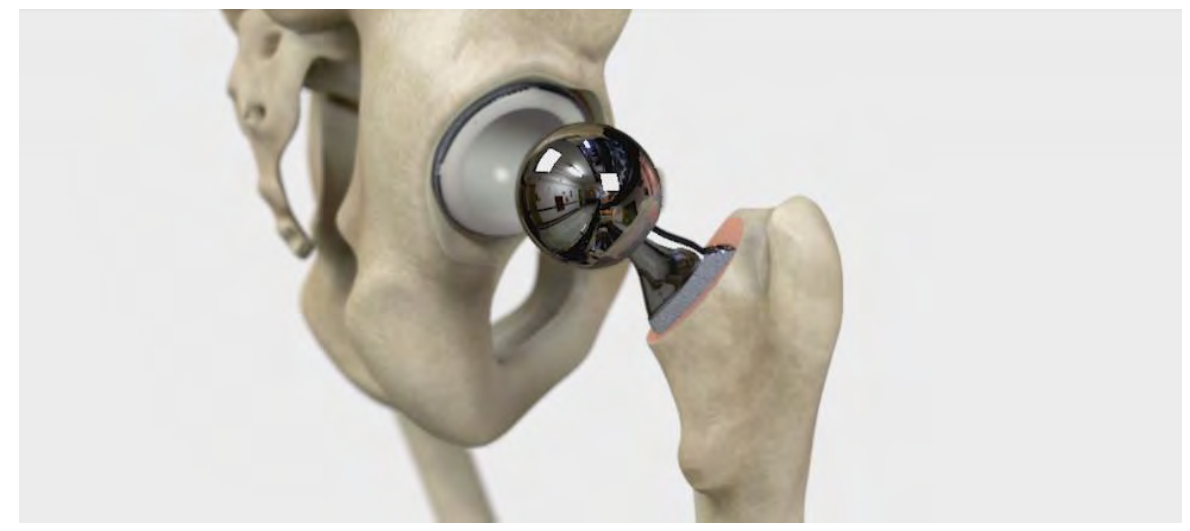

Figure 4. The last version of the film in the section of artificial hip component fitted to the acetabulum, after the feedback-loop comments on the film from healthcare personnel and patients.

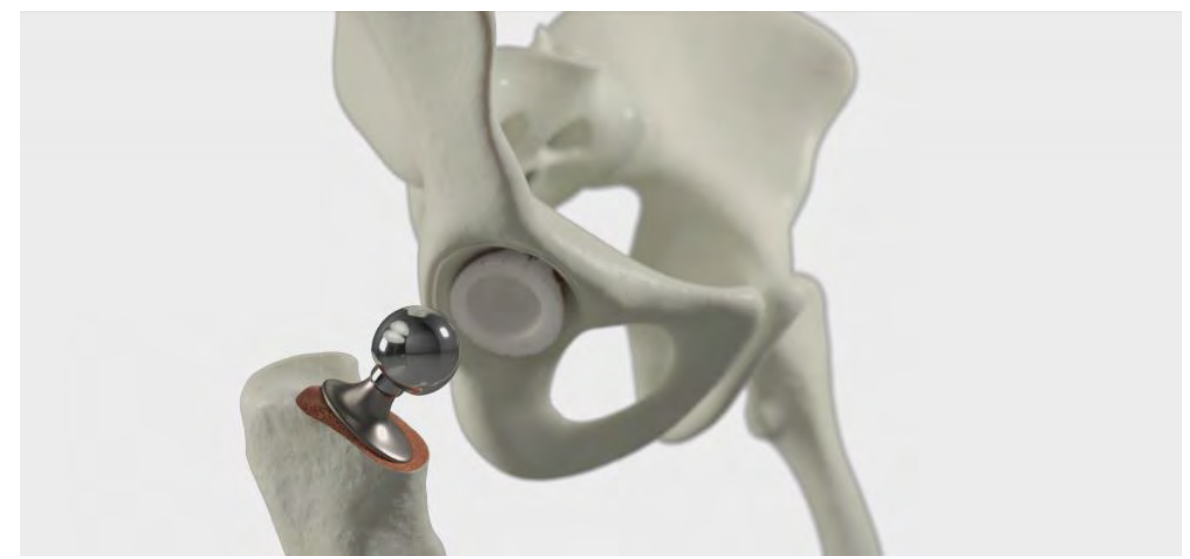




\section{Conclusion and further work}

It is important to consider each group of professionals working together in a surgical department. The differences become even more clear when implementing the film in another clinic within the same surgical profession. Each department is often keen to change various details in the manuscript to reflect their specific workflow.

Healthcare professionals are often eager to give feedback. However, it seems that different professions give different kinds of feedback, taking pride in their own specific profession not always seeing the full picture. It is important to consider every branch of healthcare professionals since their expertise need to be conveyed in the film. During the different design cycles, it became the 3D-animators responsibility to limit the amount of information and produce a well-balanced film containing all aspects of the patients' journey. The film will continue to evolve, even with other clinics as clients, and it will also be adapted and adjusted in order to their feedback.

\section{Acknowledgements}

This research received external funding by Interreg Sweden-Norway, European Regional Development Fund (ERDF) (grant number: 20202391).

\section{References}

Baskerville, R., Baiyere, A., Gregor, S., Hevner, A., \& Rossi, M. (2018). Design science research contributions: finding a balance between artifact and theory. Journal of the Association for Information Systems, 19(5), 3.

Hamel, M. B., Toth, M., Legedza, A., \& Rosen, M. P. (2008). Joint replacement surgery in elderly patients with severe osteoarthritis of the hip or knee: decision making, postoperative recovery, and clinical outcomes. Archives of internal medicine, 168(13), 1430-1440.

Hevner, A. R. (2007). A three cycle view of design science research. Scandinavian journal of information systems, 19(2), 4.

Hevner, A. R., March, S. T., Park, J., \& Ram, S. (2004). Design science in information systems research. MIS quarterly, 75-105.

Hevner, A., \& Chatterjee, S. (2010). Design science research in information systems. In Design research in information systems (pp. 9-22). Springer, Boston, MA.

Kessels, R. P. (2003). Patients' memory for medical information. Journal of the Royal Society of Medicine, 96(5), 219-222.

Lawford, B. J., Bennell, K. L., Kasza, J., \& Hinman, R. S. (2018). Physical Therapists' Perceptions of Telephone and Internet Video-Mediated Service Models for Exercise Management of People with Osteoarthritis. Arthritis care \& research, 70(3), 398-408.

Palazzo, C., Nguyen, C., Lefevre-Colau, M. M., Rannou, F., \& Poiraudeau, S. (2016). Risk factors and burden of osteoarthritis. Annals of physical and rehabilitation medicine, 59(3), 134-138.

Uesugi, Y., Koyanagi, J., Takagi, K., Yamaguchi, R., Hayashi, S., \& Nishii, T. (2018). Exercise Therapy Interventions in Patients with Hip Osteoarthritis: Comparison of the Effects of DVD and Website-Based Interventions. JMIR rehabilitation and assistive technologies, 5(1), e1. 